Un precursor desconocido de la sintaxis estructural: H. Tiktin, por Eugenio Coseriu

\title{
UN PRECURSOR DESCONOCIDO DE LA SINTAXIS ESTRUCTURAL: H. TIKTIN, POR EUGENIO COSERIU ${ }^{1}$
}

\author{
Eugenio Coseriu (1921-2002) \\ Seminario de Lenguas Románicas \\ Universidad de Tubinga (Alemania) \\ Artículo traducido del francés por Jorge Mauricio Molina Mejía ${ }^{2}$ y Maribel Betancur Serna ${ }^{3}$
}

Recibida: 05/06/2020 - Aprobada: 17/08/2020 - Publicada: 15/04/2021

DOI: doi.org/10.17533/udea.lyl.n79a30

Resumen: Se ofrece una traducción inédita de uno de los trabajos de Eugenio Coseriu (1980). Se trata de un capítulo historiográfico que busca escudriñar los esquemas de representación sintáctica de las oraciones, denominados stemmas. Se puede, así, vislumbrar todo un trabajo de rastreo histórico que busca remitirse hasta los antecesores de este tipo de modelos sintácticos, atribuidos a Tesnière (1934) y a algunos estructuralistas norteamericanos como Bloomfield, Nida y Hockett a partir de 1940. Coseriu presentaría entonces a Heimann Hariton Tiktin, un filólogo alemán, quién habría propuesto en 1883 un tipo de representación fundamentado en un modelo de dependencias.

Palabras clave: sintaxis estructural; representación sintáctica; stemmas; constituyentes inmediatos; modelo de dependencias.

\section{AN UNKNOWN PRECURSOR TO STRUCTURAL SYNTAX: H. TIKTIN, BY EUGENIO COSERIU}

\begin{abstract}
An unpublished translation of one of the works of Eugenio Coseriu (1980) is offered. It is a historiographic chapter that seeks to scrutinize the syntactic representation schemes of sentences, called stemmas. One can thus glimpse a whole work of historical tracing that seeks to refer to the predecessors of this type of syntactic models, attributed to Tesnière (1934) and to some American structuralists such as Bloomfield, Nida and Hockett from 1940. Coseriu would then present Heimann Hariton Tiktin, a German philologist, who would have proposed in 1883 a type of representation based on a model of dependencies.
\end{abstract}

Key words: structural syntax; syntactic representation; stemmas; immediate constituents; dependencies model.

1. Nota aclaratoria: En este trabajo se aborda la traducción inédita de un artículo escrito por Eugenio Coseriu (1921-2002) en 1980 y que lleva por título Un précurseur méconnu de la syntaxe structurale: H. Tiktin; trabajo publicado en una edición especial de Recherches de Linguistique : Hommages à Maurice Leroy (1980), publicado bajo el sello de las Editions de l'Université de Bruxelles. Esta editorial publica, generalmente, bajo la modalidad de Open Access (Acceso abierto), por lo cual se realizó un cuidadoso trabajo de traducción que tiene en cuenta las especificidades del texto original, y que no se desvía del legado dejado por el lingüista de origen rumano; siempre citando adecuadamente la fuente original del texto. Por otra parte, es importante constatar que el texto original también se encuentra bajo la modalidad de libre acceso en el Eugenio Coseriu Archiv (Archivo Eugenio Coseriu) de la Universidad de Tubinga (Alemania). http://www.coseriu.de/

2. Docente titular de la Facultad de Comunicaciones y Filología de la Universidad de Antioquia (Colombia). Coordinador del Grupo de Estudios Sociolingüísticos y del semillero de investigación Corpus Ex Machina, ambos adscritos a la Facultad de Comunicaciones y Filología de la Universidad de Antioquia. Correo electrónico:jorge.molina@udea.edu.co

3. Estudiante del pregrado de la Licenciatura en Literatura y Lengua Castellana de la Facultad de Educación de la Universidad de Antioquia (Colombia). Miembro del semillero de investigación Corpus Ex Machina, adscrito a la Facultad de Comunicaciones y Filología de la Universidad de Antioquia (Colombia). Correo electrónico: maribel.betancurs@udea.edu.co

Editores 


\section{Preámbulo}

$\mathrm{E}$

n el campo de la sintaxis la utilización de esquemas o de árboles de representación ha sido una constante desde comienzos del siglo xx hasta la actualidad (Coseriu, 1980; Hudson, 2014; Mazziotta \& Kahane, 2017); por lo menos, en lo que corresponde a los modelos basados ya sea en la gramática de dependencias de Lucien Tesnière o de constituyentes inmediatos (que se les atribuyen, notablemente, a los estructuralistas norteamericanos). El modelo propuesto por Tesnière ha sido considerado como un modelo representativo en lo que concierne a la sintaxis de tipo estructural (Sarfati \& Paveau, 2014). No obstante, un artículo propuesto por Eugenio Coseriu en 1980, dentro de una obra en homenaje a Maurice Leroy, y que lleva por título Un précurseur méconnu de la syntaxe structurale : H. Tiktin, presenta, a partir de un trabajo historiográfico, la posibilidad de que este tipo de análisis fuera muy anterior a la obra del lingüista francés.

El particular interés, en esta propuesta de Coseriu, nace de la posibilidad de proveer a los estudiosos y a las personas interesadas en el campo de la sintaxis, el acceso a esta clase de información; que hasta ahora estaba en francés. Por lo cual, se determinó hacer lo más legible posible el artículo en cuestión para el público hispanohablante. Con tal fin, se hizo necesario modificar algunos de los muchos incisos empleados por el autor, en vista de que se busca una lectura mucho más fluida; sin por ello menoscabar el sentido original expresado por el lingüista de origen rumano.

En lo que respecta al proceso de traducción per se, se decidió traducir todo lo que estaba en francés incluyendo las oraciones de ejemplo, las cuales provenían de interpretaciones efectuadas por el autor, a partir de oraciones en rumano originarias de Tiktin. En ese sentido, era importante partir de una traducción al español que mantuviera el carácter original de tales oraciones. Por tal motivo, se empleó el formato de pie de página, con la mención «N. del T.», es decir, anotaciones realizadas por los traductores, con el fin de posibilitar su comprensión. Por lo demás, se determinó traducir al español las demás oraciones que estaban en otras lenguas como el inglés, el alemán, el sueco y el latín; para las cuales Coseriu no proponía una traducción al francés.

En lo que concierne a la estructura del artículo, en primer lugar, se optó por conservar la numeración original propuesta por Coseriu, puesto que esta sirvió de referencia en los diferentes reenvíos del autor. En segundo lugar, en lo referente a los stemmas y a los árboles de constituyentes inmediatos, y a cualquier otra representación esquemática, estas se trataron con toda la rigurosidad del caso y se mantuvieron en la lengua original en la que aparecían en el artículo y, además, se tuvo en cuenta el hecho de que carecían de un título - aunque el autor siempre citó las fuentes-, y se preservó esa idea. El hecho de guardar la lengua original en los esquemas parte de dos aspectos importantes: a) al intentar traducir los elementos del esquema es muy factible que los elementos gramaticales no correspondan a las versiones originales y que algunos de ellos no encajen con respecto a la versión original; y b) en el texto original el propio Coseriu tampoco los traduce al francés - cuando se trata de oraciones en rumano, alemán, latín y sueco- - justamente debido al tema de la incompatibilidad entre árboles en las lenguas originales y su versión en la lengua de escritura del artículo. 
Un precursor desconocido de la sintaxis estructural: H. Tiktin, por Eugenio Coseriu

Por otro lado, en el caso particular en el que los esquemas sintácticos aparecían dentro de un pie de página, y que por motivos de forma no era posible ubicarlos allí; se optó por agregarlos en el cuerpo del texto, en la zona en donde aparecía el número que lo indicaba. En tercer lugar, en relación con la bibliografía, Coseriu no estableció una en la parte final del artículo, puesto que va nombrando todas las obras y sus respectivos autores a medida que va desarrollando el tema en cuestión; en este orden de ideas, se recopiló la lista de autores citados tanto por el lingüista rumano, como por los traductores y se anexó en la parte ubicada al término de la obra, justo después de su firma.

Así, se considera que la importancia de este artículo y de su traducción a la lengua castellana radica en el hecho de que este tipo de trabajos puede convertirse en un insumo importante, en lo que respecta a la historiografía de la sintaxis y del análisis a partir de las gramáticas en él presentadas. Y para esto se hizo énfasis en tres aspectos importantes:

En primer lugar, hay que reconocer que esta traducción nace de un interés de los traductores por la sintaxis y, muy particularmente, frente a los modelos de representación sintáctica, bien sean esquemas, diagramas, stemmas, árboles de constituyentes inmediatos, etc. En este sentido, ante el trabajo propuesto por Coseriu (1980), el objetivo primordial se centró en su traducción y su difusión entre los especialistas en el campo de la lingüística interesados en esta temática.

En segundo lugar, se debe tener claro que, en lo que concierne a la lengua española, los trabajos de índole historiográfica en el campo de la sintaxis son más bien escasos, razón por la cual la traducción propuesta tiene un valor agregado. Por ende, luego de un proceso de búsqueda de material historiográfico en la sintaxis, se halló que la mayor parte de artículos y de obras que abordan la problemática en cuestión se encuentran particularmente en inglés y en francés (Hudson, 2014; Imrenyi \& Mazziotta, 2020, Mazziotta \& Kahane, 2017; Osborne, 2019), lo que evidencia el gran vacío de producción intelectual que dicha área del conocimiento tiene en lengua española.

En tercera y última instancia, es posible que muchos estudiosos del lenguaje partan de una concepción, tal vez un poco amañada, según la cual, se considere que tanto Tesnière (1934, 1959), como los estructuralistas norteamericanos — desde los años 1940 — han sido los primeros en proponer los esquemas de representación sintáctica; vale decir no solo el modelo denominado gramática de dependencias — sintaxis estructural—, sino también el de constituyentes inmediatos — antecesora de la Gramática Generativa Transformacional—. Como se pudo observar, a lo largo del estudio realizado por Coseriu, es sumamente interesante el poder constatar que desde comienzos del siglo XIX — véase en 6.3, la gráfica propuesta por Coseriu, en la que el autor propone un árbol genealógico que va desde Billroth (1832), hasta Tesnière (1934)—, ya se habían creado esquemas y diagramas de carácter sintáctico, con el fin de enseñar diversas lenguas.

\section{Traducción del artículo original (Coseriu, 1980)}

1.1. Lucien Tesnière afirma en su obra Éléments de syntaxe structurale, París (1959, p. 15), que la idea de 
Un precursor desconocido de la sintaxis estructural: H. Tiktin, por Eugenio Coseriu

los «stemmas», es decir, de la representación gráfica de las conexiones sintácticas, le surgió en junio de 1932. Es una idea que aplicó por primera vez, poco tiempo después, en su artículo Comment construire une syntaxe, Bull. de la Fac. des Lettres de Strasbourg (1934), y luego en su Esquisse d'une syntaxe structurale, París (1953). No fue sino más tarde (en el año de 1936), agrega, que encontró unos stemmas en la obra Učebnaja kniga po russkomu jazyku, Moscú-Leningrado (1929), de Ušakov, Smirnova y Ščeptova. Y se enteró de que Barchudarov y Princip (alumnos de Ščerba) también los habían empleado a partir de 1930. Tesnière observa, sin embargo, que los stemmas de estos lingüistas — que, por lo demás, no empleaban más que ocasionalmente y a manera de ejemplo — no corresponden a los suyos, ya que estos se basan en la división de la oración en sujeto y predicado, lo que considera como gramaticalmente incorrecto. En todo caso, la fecha más antigua en la cual se habría empleado esquemas sintácticos sería, según las informaciones dadas por Tesnière, en 1929.

1.2. En efecto, en la lingüística americana de ascendencia bloomfieldiana, los esquemas sintácticos (sobre todo arbóreos) empleados para el análisis en «constituyentes inmediatos», esquemas demasiado comunes en la actualidad y que pueden encontrarse en casi todos los manuales de lingüística descriptiva, no salen a la luz sino hasta veinte años más tarde. El mismo Bloomfield, Language, New York (1933, p. 161), habla de «constituyentes inmediatos» sin emplear los stemmas, y el famoso artículo de R. S. Wells, Immediate Constituents, Language 23 (1947, pp. 81-117), tampoco contiene ninguno. El primero en emplear «árboles» sintácticos en el marco del estructuralismo norteamericano parece haber sido Eugene Nida, en su Morphology. The Descriptive Analysis of Words, Ann Arbor (1946, 2 a edición de 1949, pp. 87, 101, 105). Luego, este tipo de esquemas, un poco modificados por Ch. C. Fries, The Structure of English, New York (1952, pp. 264-273), se expande y se hace «popular» sobre todo gracias a H. A. Gleason, An Introduction to Descriptive Linguistics, New York (1955, pp. 129-131), y a Ch. F. Hockett, A Course in Modern Linguistics, New York (1958, pp. 152-155, 158-161, 178, 180, 188); en el que los mismos esquemas son posteriormente elaborados y presentados bajo diferentes formas. Por otra parte, los stemmas utilizados en el análisis de «constituyentes inmediatos», así como los «phrase markers» ${ }^{4}$ de la gramática transformacional que le corresponden (y derivan de aquellos), no son tampoco del mismo tipo de los de Tesnière, dado que estos provienen de otra concepción de relaciones sintácticas: al igual que los stemmas de los gramáticos soviéticos ya mencionados por el lingüista francés, estos se apoyan, en principio, en la división binaria de la oración en sujeto y predicado; además, son esquemas de «combinación» (o «adición»), no de «dependencia».

1.3. Se sabe, sin embargo, que esquemas análogos fueron empleados en lingüística mucho antes de 1929. O. Jespersen, Analytic Syntax, Copenhague (1937, p. 93), reproduce un esquema bastante complejo de una oración inglesa propuesto por Isabel Fry, A Key to Language, Londres (1925, p. 64). Y, M. B. Collinder, Noam Chomsky und die generative Grammatik. Eine kritische Betrachtung, Uppsala (1970, p. 6), señala que el gramático sueco N. Beckman emplea los «Stammbäume» sintácticos en su Svensk språklära för den högre undervisningen [sic] $]^{5}$ 4. «Árboles sintácticos» en la teoría chomskiana. (N. del T.).

5. En el presente artículo (Coseriu, 1980) aparecen dos obras atribuidas a un mismo autor, N. Beckman: la primera con el nombre de: Svensk språklära för den högre undervisningen de 1904, y la segunda: Svensk språklära för den högre elementarundervisningen de 1935. No obstante, y luego de una investigación que tenía como finalidad establecer la correcta bibliografía del artículo, se pudo establecer que, en realidad, se trata de la misma obra, pero de dos ediciones diferentes y con una variación en el nombre. En ambos casos, el nombre encontrado para las dos obras corresponde, más bien, al de elementarundervisningen, y no al de undervisningen. Sin embargo, se prefirió guardar la versión original propuesta por Coseriu, con la anotación «sic» (textualmente). (N. del T.). 
Un precursor desconocido de la sintaxis estructural: H. Tiktin, por Eugenio Coseriu (1904), y que a su vez Beckman nos conduce a F. Kern, Zur Methodik deutschen Unterrichts (1883).

2.1. Por el contrario, lo que parece ser escapó a los conocedores de la historia de la lingüística, así como a los lingüistas que se ocupan de la sintaxis estructural ${ }^{6}$, es que ya en 1893 el romanista de origen germano-rumano Hariton (Heymann) Tiktin (1850-1936) había empleado en gran medida stemmas exactamente del mismo tipo de los de Tesnière, y que poseían la misma concepción de la estructura de la oración, como puede apreciarse en su segundo volumen (dedicado a la sintaxis) de su obra Gramatica română, Iaşi (1891-1893). En el $\S 445$ de esta obra (pp. 125-129) Tiktin propone principalmente con el objetivo de hacer evidente la estructura de una oración analizada («Spre a ne face o idee lămurită de structura unei propoziţiuni analizate»), lo que él llama la «síntesis figurada» («sinteza figurată»); es decir, precisamente, la representación gráfica de las conexiones y determinaciones al interior de cada oración ${ }^{7}$. Al mismo tiempo, la «síntesis figurada» debería ser una instancia de control para la exactitud del análisis («... este totodată şi controlul cel mai bun pentru exactitatea analizei»).

2.2. Para Tiktin el punto de partida para este procedimiento es el verbo conjugado (predicado verbal), que sitúa en la parte superior y que une por medio de líneas, en primer lugar, al sujeto y — si existe uno- al complemento de objeto; situados por debajo del verbo, respectivamente a su izquierda y derecha, así como a otros complementos eventuales, estos también situados, por lo general, más abajo y a la derecha. No obstante, si existen varios complementos del mismo nivel estructural, es decir que hacen referencia (según la doctrina gramatical más usada) directamente al predicado verbal; estos se encontrarán situados «en niveles» a la derecha del verbo conjugado. Las eventuales determinaciones posteriores del sujeto y de los núcleos de los complementos se encuentran situadas, a su vez, por debajo de aquellos; mientras que la negación está ubicada inmediatamente bajo el verbo. Así Tiktin representa los proverbios Buturuga mică răstoarnă carul mare («La petite souche renverse le grand char» $\left.{ }^{8}\right)^{9}$, Corb la corb nu scoate ochii («Corbeau à corbeau n'arrache pas les yeux» ${ }^{10}$ ) y Din coadă de cîine sită de mătase nu se face («De queue de chien on ne fait pas de tamis de soie» ${ }^{11}$ ), de la siguiente manera:

6. E incluso, el autor de un libro sobre «el hombre y la obra»: I. Rizescu, H. Tiktin, Omul şi opera, Bucarest (1971).

7. En la $2^{\mathrm{a}}$ edición, Bucarest (1895, reimpreso en 1902 y 1905), esta «síntesis» figura en las páginas $107-110$ (§ 457); en la $3^{\mathrm{a}}$ (en un solo volumen), publicado por I. A. Candrea, Bucarest (1945), en las páginas 204-207 (§ 456). Citamos aquí a partir de esta última edición.

8. «El pequeño tronco volteó al gran carro». (N. del T.).

9. $\quad$ Para que se pueda apreciar exactamente el valor y el sentido de los análisis y de los diagramas de Tiktin, traduciremos sus ejemplos tan literalmente como sea posible, yendo en ocasiones a los límites de la aceptabilidad en francés (por ejemplo, en lo que concierne al orden de las palabras y al empleo del artículo).

10. «Cuervo a cuervo no le saca los ojos». (N. del T.).

11. «De la cola del perro no se hace un tamiz de seda». (N. del T.). 


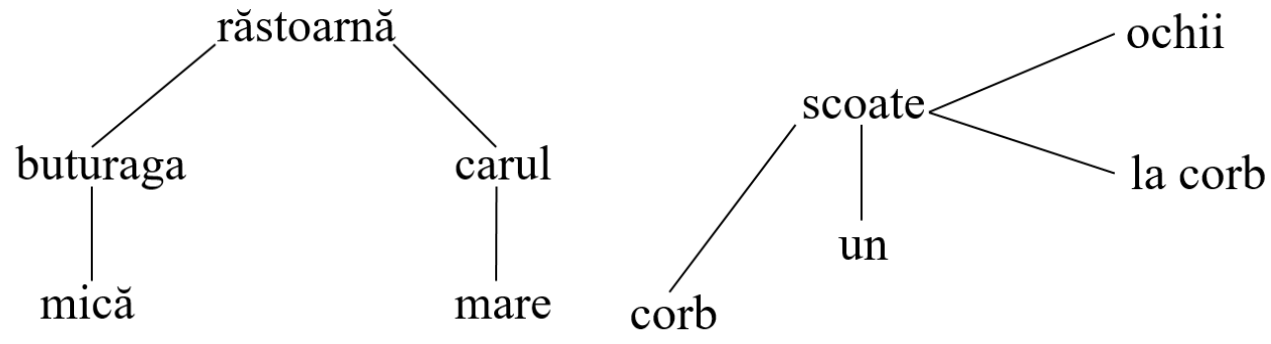

Y:

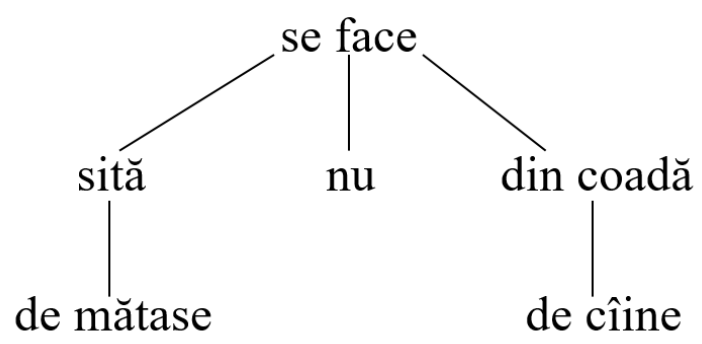

Los elementos no expresados pero implícitos en la oración son ubicados en los lugares que les corresponden en el stemma, entre paréntesis ( ). Así, en el caso de Schimbarea domnilor, bucuria nebunilor («Le changement des princes, [c'est] la joie de fous» ${ }^{12}$ ) y de Umblă după potcoave de cai morţi («[Il] va cherchant des fers à cheval de chevaux morts» $\left.{ }^{13}\right)$ :

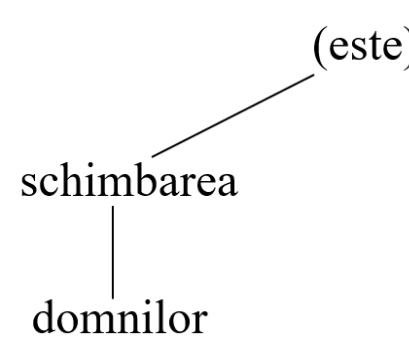

domnilor

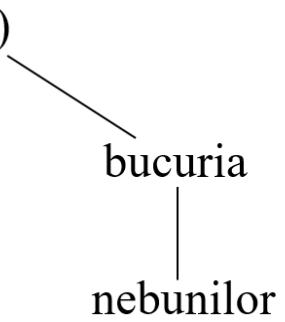

(el)

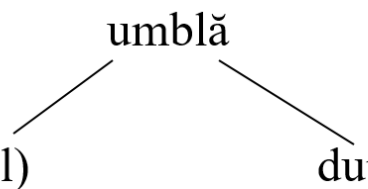

En cuanto a los elementos «múltiples» (coordinados), estos son tratados, en cada caso, como una sola unidad y situados sobre la misma línea. Así, por ejemplo, en lo referente a O linişte îngrozitoare înneacă şi codru şi castel («Un silence effrayant étouffe forêt et château» ${ }^{14}$ ):

12. «El cambio de los príncipes, [es] la alegría de los locos». (N. del T.).

13. «[Él] va buscando herraduras de caballos muertos». (N. del T.).

14. «Un silencio aterrador asfixia bosque y castillo». (N. del T.). 


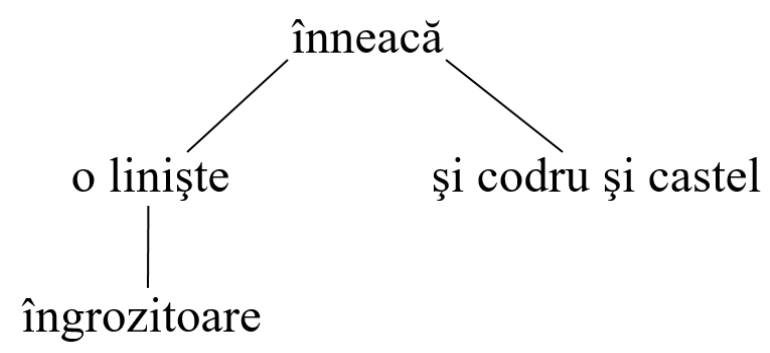

Finalmente, las determinaciones que conciernen a construcciones enteras son unidas a aquellas otras por medio de llaves \{\}. Cf. Aceşti boieri erau bine văzuţi la curtea lui Vodă («Ces boyards étaient bien vus à la cour du prince ${ }^{15}$ ) y Muşcătura şarpelui cu clopoţei din America ucide pe omul cel mai viguros în cîteva minute («La morsure du serpent à sonnettes de l'Amérique tue l'homme le plus vigoureux en quelques minutes») ${ }^{16}$ ):

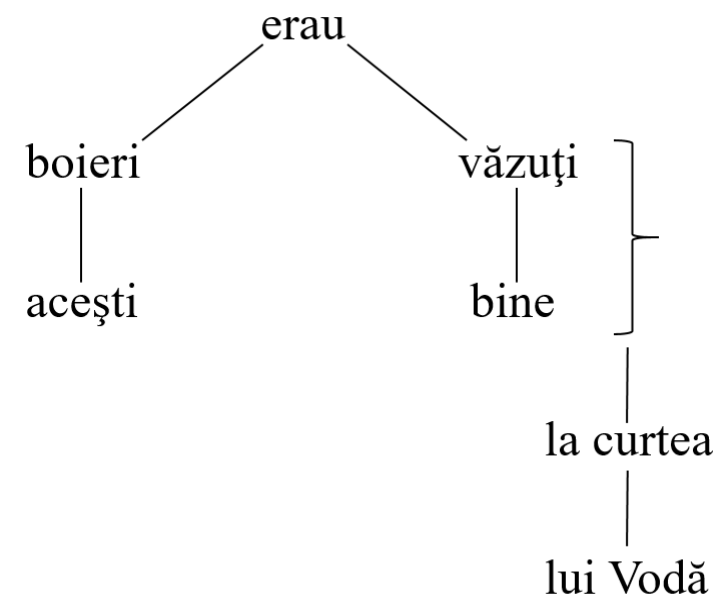

Y:

15. «Estos boyardos eran muy bien vistos en la corte del príncipe». El término boyardo hace referencia a los aristócratas en los países eslavos. (N. del T.).

16. «La mordedura de la serpiente cascabel de América mata al hombre más vigoroso en pocos minutos». (N. del T.). 


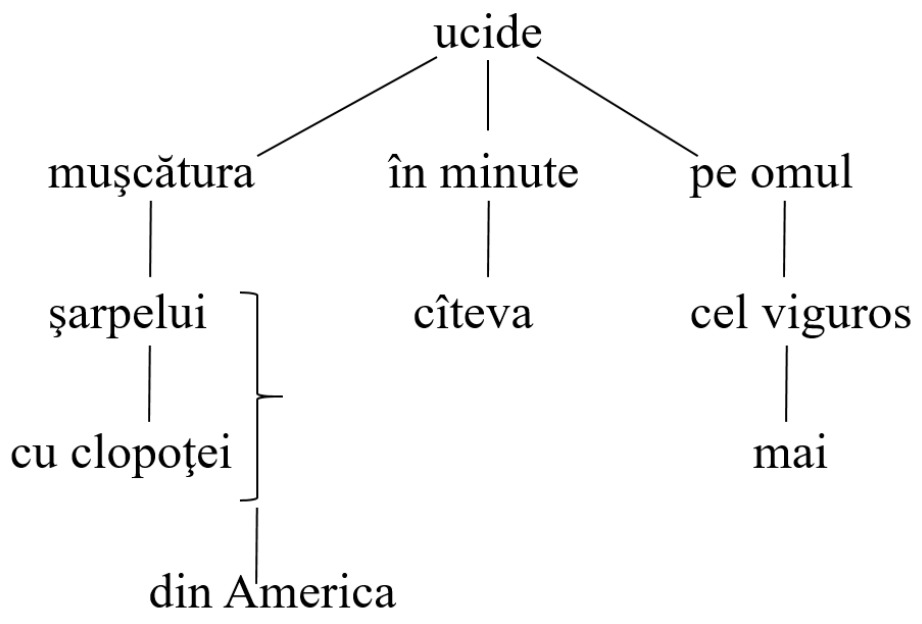

2.3. Este mismo tipo de análisis y de representación gráfica, Tiktin lo aplica también en el caso de la oración compleja (que contiene proposiciones subordinadas o «equivalentes de subordinadas»). En la «síntesis figurada», de una de tales oraciones, las proposiciones subordinadas o las construcciones que equivalen a estas ${ }^{17}$ —enteramente reproducidas, o bien indicadas por su primera palabra o por unas letras - se encuentran primero que todo situadas en los lugares de las funciones que poseen con respecto a la proposición principal (sujeto, complemento de objeto, complemento circunstancial, etc.), y luego cada una de ellas es representada por diagramas análogos a los de las oraciones simples (op. cit., § 518, pp. 242-24318; f. también las instrucciones que se refieren a los procedimientos a aplicar en este caso, pp. 218-219).

3.0. Como podemos observar la analogía es casi perfecta — en la concepción gramatical subyacente o explícita, y a menudo incluso en los detalles — entre el análisis («síntesis») de Tiktin y el de Tesnière.

3.1. Para los dos autores la idea principal es la idea del orden «de régimen» - «regido», con el verbo conjugado como regente principal. Cf. por ejemplo en Tesnière, Éléments (1959, p. 61), (Alfred a oublié hier son chapeau $)^{19}$ :

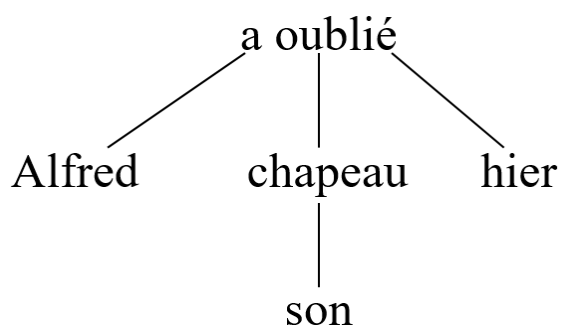

17. Tiktin considera como sucedáneas de subordinadas («substituiri de dependente») las construcciones con el gerundio y con el infinitivo así como la aposición (Gramatica română, 1891-1893, p. 224).

18. 1a edición, II, pp. 145-146; 2a edición, II, pp. 124-125.

19. «Alfredo olvidó su sombrero ayer». (N. del T.). 
Ambos autores sitúan, en cada caso, el determinante bajo el determinado; además, estos separan desde el comienzo los diferentes «complementos» del verbo y ubican las preposiciones con el sustantivo que aquellas rigen y los auxiliares con el verbo «principal». Tanto para Tesnière como para Tiktin, los elementos coordinados se sitúan en la misma línea ( $c f$. Éléments, 1959, p. 80: stemma de Les hommes craignent la misère et la mort ${ }^{20}$ ) y los elementos implícitos figuran entre paréntesis ( $c f$. por ejemplo Éléments, 1959, p. 160).

3.2. Las principales diferencias entre los dos autores tienen que ver, sobre todo, con algunos detalles en la representación gráfica. Tiktin no separa los artículos ni el pronombre reflexivo de la conjugación denominada «pronominal» ${ }^{21}$; mientras que Tesnière, al mismo tiempo que ubica el artículo definido al lado del sustantivo que aquel determina, separa de este último el artículo indefinido; $\mathrm{y}$, además, separa también el pronombre reflexivo del verbo conjugado. Así, representa Les chevaux mangèrent le foin ${ }^{22}$ (p. 54) por medio del stemma:

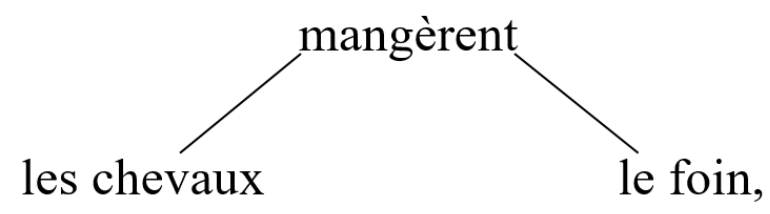

Pero, un dîner léger ${ }^{23}$ (p. 63), Votre jolie cousine chante une chanson ${ }^{24}$ (p. 145) y Alfred se tue ch $^{25}$ ( 241) por medio de:

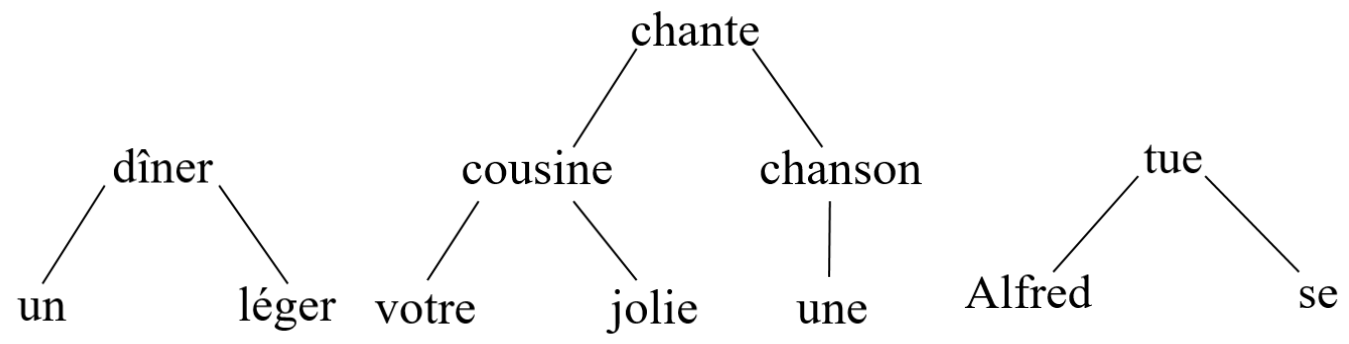

20. «Los hombres temen la miseria y la muerte». (N. del T.).

21. Tiktin declara explícitamente (op. cit., p. 204) que los artículos (tanto el «definido» —enclítico en rumano-como el indefinido y el adjetival), las preposiciones, los verbos auxiliares, los pronombres de la voz «reflexiva» y la partícula să del subjuntivo deben ser consideradas en el análisis como constituyentes de una sola palabra [gramatical] con la palabra [lexical] que determinan.

22. Los caballos comieron el heno». (N. del T.).

23. «Una cena ligera». (N. del T.).

24. «Su hermosa prima canta una canción». (N. del T.).

25. «Alfred se mata». (N. del T.). 
El predicado nominal, Tesnière lo sitúa en ocasiones con la cópula y a veces lo separa de esta. $C f$. los stemmas de La maison est neuve ${ }^{26}$ (p. 160) y de Ce livre est très beau ${ }^{27}$ (p. 181)
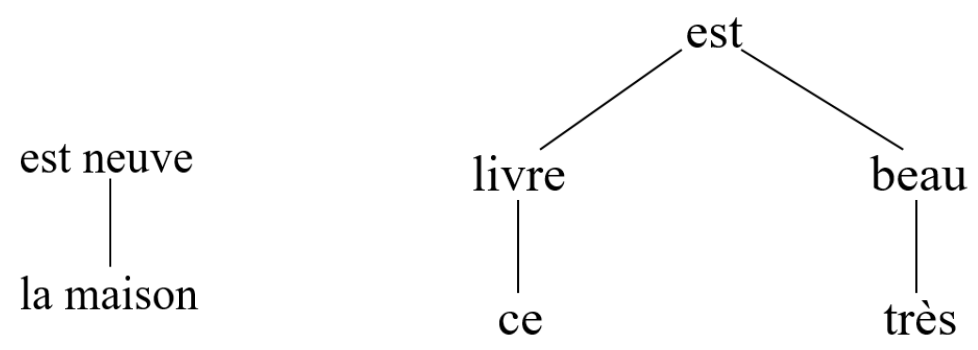

La negación, por su parte, es presentada por Tiktin como si fuera una determinación externa al verbo, mientras que Tesnière la ubica al lado de aquel; al menos en una primera fase de su análisis verbal. Así, en el caso de Alfred ne chante $\operatorname{pas}^{29}$ (p. 218), se representa a través del stemma:

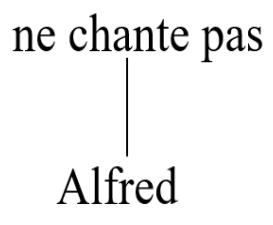

Pero ya en su obra Esquisse (1953, pp. 6-7), Tesnière distingue la negación «nuclear» (tal como se encuentra, por ejemplo, en Personne ne chante ${ }^{30}$, Alfred ne fait rien ${ }^{31}$ ) y la negación «conexional», y presenta esta última de una forma muy adecuada, como la que hace referencia a la misma conexión entre el verbo y el sujeto ${ }^{32}$ :

\footnotetext{
26. «La casa es nueva». (N. del T.).

27. «Este libro es muy hermoso». (N. del T.).

28. Estos dos stemmas pueden, sin embargo, ser considerados como correspondientes a dos fases sucesivas del mismo análisis.

29. «Alfred no canta». (N. del T.).

30. «Nadie canta». (N. del T.).

31. «Alfred no hace nada». (N. del T.).

32. En Éléments (1959, p. 218), lo que se dice en el texto a propósito de la negación «conexional» justificaría también este mismo stemma, más bien que el que allí figura.
} 


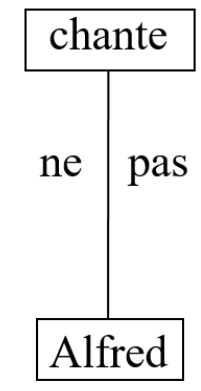

No encontramos en Tesnière un stemma que corresponda exactamente al tipo şarpele cu coploţei din America, en donde America no determina şarpele ni coploţei, sino el sintagma completo şarpele cu coploţei ${ }^{33}$. Es posible, sin embargo, suponer que habría presentado tal construcción bajo la forma:

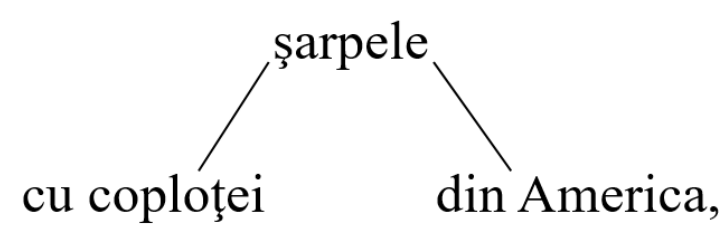

Como lo hace en el caso de $\mathrm{de}^{34}$ :

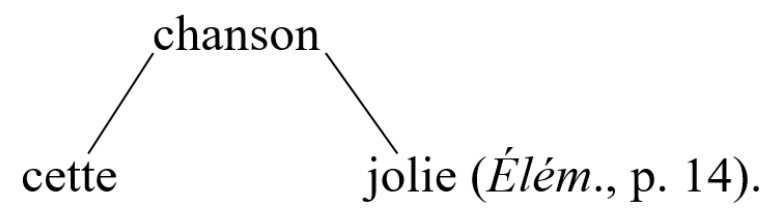

Para Tiktin, por el contrario, esta última construcción habría sido probablemente:

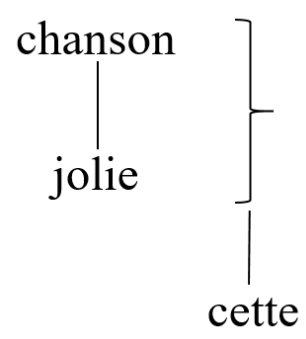

33. «La serpiente [de] cascabel de América».

34. «Esta hermosa canción». $(\mathrm{N}$ del $\mathrm{T})$. 
Igualmente, la oración Alfred fourre toujours son nez partout ${ }^{35}$, que Tesnière en Éléments (1959, p. 103), representa con el stemma:

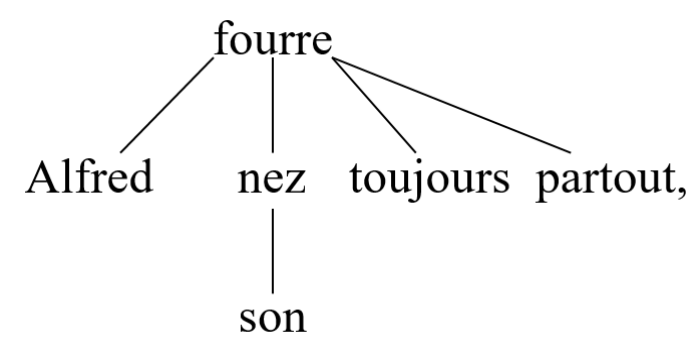

Habría sido para Tiktin, más bien:

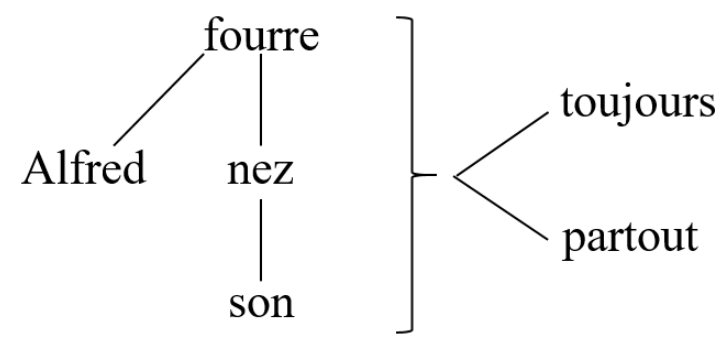

Pero, Tesnière también considera la posibilidad de la determinación de un sintagma completo, y de una representación gráfica que le corresponda. Así, por ejemplo, en el caso de L'auto rouge que vous avez vue hier ${ }^{36}$ (Éléments, 1959, p. 154):

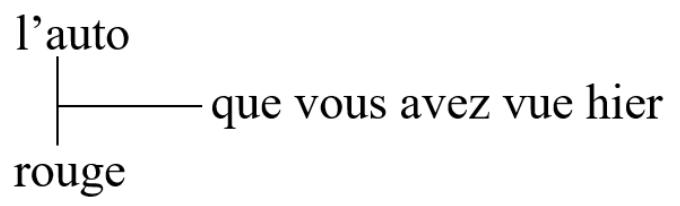

Lo que significa que también habría podido presentar şarpele cu coploței din America, bajo la forma:

35. «Alfred siempre mete su nariz en todas partes». (N. del T.).

36. «El auto rojo que usted vio ayer». (N. del T.). 


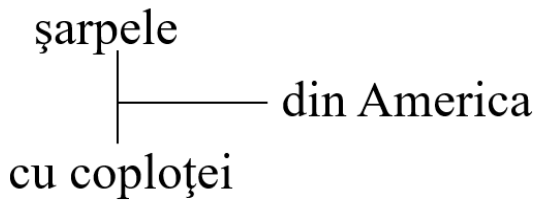

Finalmente, no se encuentra nada en el trabajo de Tiktin sobre el problema de la anáfora; problema que Tesnière trata, por el contrario, de una manera muy pertinente, Éléments (1959, pp. 85 y subsiguientes).

4.1. El criterio aplicado constantemente por Tiktin en sus análisis y en los esquemas que les corresponden es el de las conexiones sintácticas «actuales», en el sentido estricto de ese término; en otras palabras, el de las conexiones que se establecen para cada caso en cualquier oración (o tipo de oración). Es por ello que considera las construcciones más o menos fijas — es decir: las que aparecen, en principio, bajo la misma forma en cualquier oración (artículo + sustantivo, preposición + sustantivo, auxiliar + verbo principal, etc.: cf. nota 19) - como unidades sintácticamente dadas, y este autor no las analiza. Por la misma razón, el orden en el que las unidades sintácticamente combinadas figuran en sus esquemas le permite reproducir el orden funcional de la estructura sintáctica, lo cual es, a menudo, muy diferente del orden lineal; $c f$. por ejemplo, el orden de corb la corb (cuervo a cuervo), aceşti boieri (estos boyardos), cel mai viguros (el más vigoroso) en la cadena hablada y en la representación gráfica.

4.2. Y también debido a su criterio de base, el análisis sintáctico de Tiktin (como también el de Tesnière) es claramente superior al análisis en «constituyentes inmediatos», y a las técnicas analíticas que se derivan de aquellos. Esto, incluso haciendo abstracción del concepto - muy discutible - de oración como la unión de sujeto y predicado (o de «sintagma nominal» y «sintagma verbal»); concepción, en el fondo, no lingüística y que proviene más bien de la lógica y de la gramática «logicista». En el análisis de constituyentes inmediatos, las combinaciones sintácticas se representan como simples adiciones no-motivadas de elementos del mismo nivel estructural. Por el contrario, el análisis efectuado por Tiktin siendo funcionalmente motivado; por lo menos, en principio, no solamente es más rico, sino al mismo tiempo mucho más adecuado. En efecto, de los tres aspectos para tomar en consideración, en lo que respecta a las estructuras sintácticas — unidades combinadas, orden de las combinaciones y relaciones entre las unidades en cada combinación—, el análisis en constituyentes inmediatos pone en evidencia los dos primeros, mientras que ignora al tercero - las relaciones de dependencia o régimen (o bien, en el sentido contrario, de «determinación») - ; es decir, precisamente el aspecto más importante, incluso esencial, desde el punto de vista lingüístico y que es buscado, en primer lugar, en el análisis de Tiktin ${ }^{37}$.

37. Es verdad que la dirección de las determinaciones, indicada por Tiktin y por Tesnière por la posición de los términos regentes y regidos en los stemmas, se encuentra indicada por medio de flechas en los esquemas de M. E. A. Nida, A Synopsis of English Syntax (1959, 4a edición publicada por B. Elson, Norman, Oklahoma, 1964, pp. XI-LVIII). Pero se trata de un caso aislado en el marco del estructuralismo norteamericano. Por otra parte, estos esquemas no fueron agregados sino hasta 1959; el texto original, presentado como tesis doctoral en la Universidad de Michigan en 1943, no los contenía. 
Un precursor desconocido de la sintaxis estructural: H. Tiktin, por Eugenio Coseriu

Así, el stemma de la oración inglesa Old men have old wives ${ }^{38}$ sería, de acuerdo con el análisis en constituyentes inmediatos:

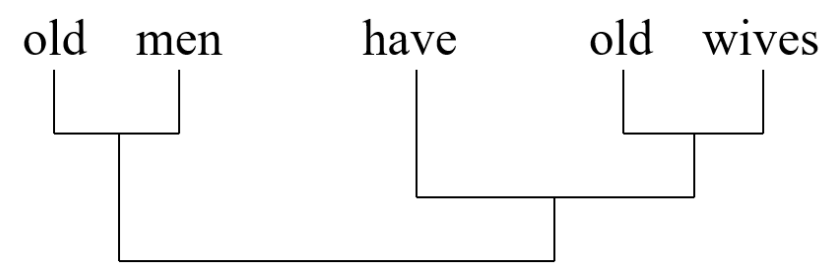

O, al revés:

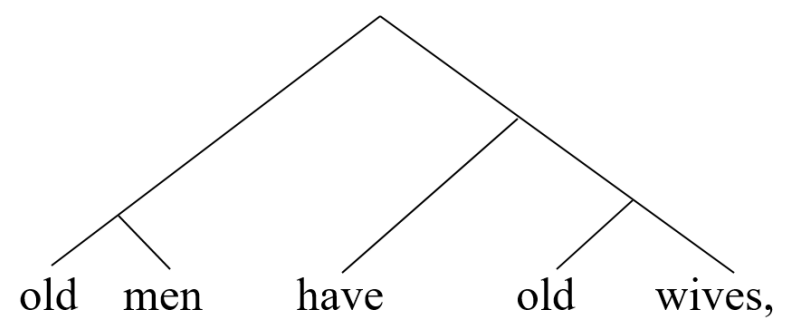

Cf. el diagrama de M. Nida de la oración Peasants throughout China work very hard ${ }^{39}$, Morphology (1949), p. $87^{40}$ :

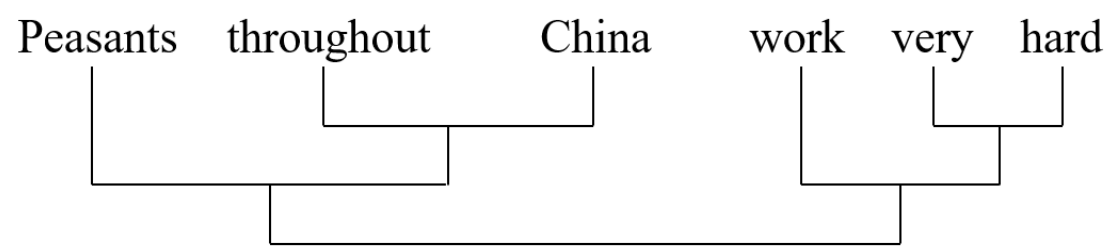

Mientras que en Tiktin el stemma referente a la oración Old men have old wives, sería:

38. «Los hombres viejos tienen esposas viejas». (N. del T.).

39. «Los campesinos a lo largo de China trabajan muy duro». (N. del T.).

40. En el artículo original este esquema aparece en un pie de página, pero por motivos de visibilidad se optó por ubicarlo a continuación del diagrama al que hace referencia. Por su parte, lo que correspondía al pie de página como tal se situó antes del diagrama. (N. del T.). 


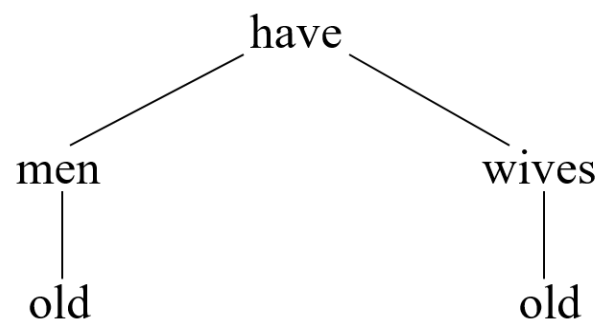

Y el stemma de la oración en rumano, citada con anterioridad: Muşcătura şarpelui cu clopoţei din America ucide pe omul cel mai viguros în cîteva minute («La mordedura de la serpiente cascabel de América mata al hombre más vigoroso en pocos minutos»), según el análisis en constituyentes inmediatos, sería simplemente:

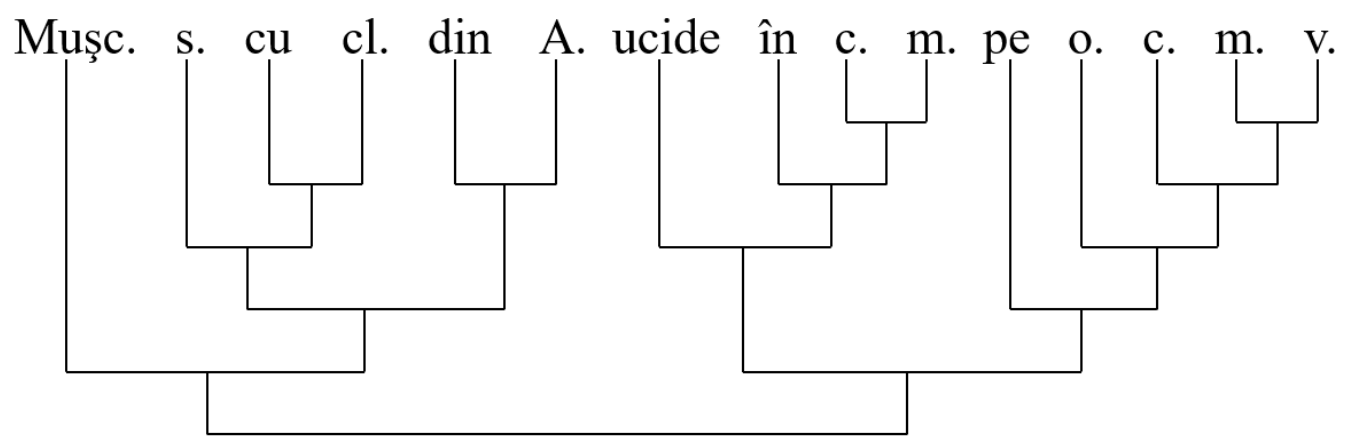

(O el mismo «árbol» invertido). Ahora bien — salvo para lo que tiene que ver con el desplazamiento de în cîteva minute- esto no sería más que una representación arbórea o en niveles de la división por medio de barras verticales (ya empleadas por R. S. Wells en su artículo de 1947). En efecto, en el análisis de constituyentes inmediatos no se trata verdaderamente de «estructuración», ni de análisis sintáctico propiamente dicho, sino más bien de «segmentación» de oraciones.

4.3. Esto no significa, de ninguna manera, que todo sea irreprochable en los análisis de Tiktin. Así, según su criterio más o menos explícito, él habría debido considerar şarpele cu coploţei como una unidad sintácticamente dada y no como conexión «actual» (no se trata de una «serpent avec des clochettes» sino de una «serpent-àsonnettes») $)^{41}$; y bine văzuţi es también una unidad lexical (no es lo mismo que văzuţi bine). Así mismo, no se sabe muy bien por qué Tiktin no adopta:

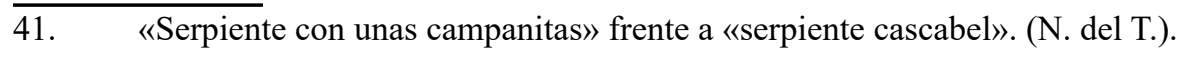




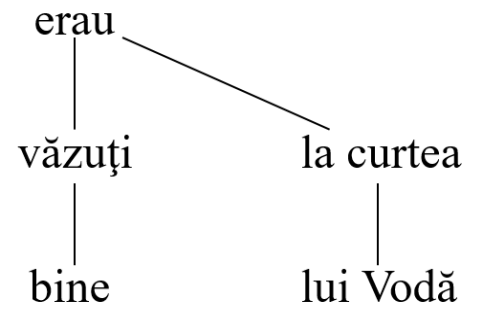

O, si se acepta su análisis ( $c f$. arriba, 2.2), no se entiende por qué no lo analiza de la misma forma:

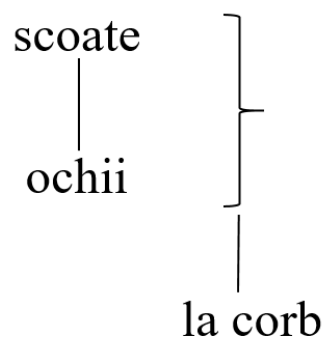

Y no se puede estar de acuerdo con su tratamiento de la negación, interpretado como determinación externa (¿«complemento»?) del verbo.

Pero lo que importa, no es la exactitud de su análisis para cada caso en particular. Es, más bien, el criterio que el autor aplica allí y consiste, sobre todo, en el procedimiento que emplea para la representación gráfica de las estructuras sintácticas; criterio y procedimiento que no eran del todo habituales para su época, y que hacen de él uno de los precursores más importantes de la sintaxis estructural de nuestro tiempo.

5.1. Sin embargo, como se ha visto, Tiktin no atribuye ninguna importancia teórica a sus diagramas: los presenta, más bien, como simples expedientes didácticos. Más aún: tampoco les atribuye el mérito de la novedad y no pretende ser el primero en emplearlos. En ese sentido, Tiktin dice muy simplemente: «Para hacer evidente la estructura de una oración analizada, empleamos [= se emplea] la sintesis figurada», como si se tratara de un procedimiento ya conocido. Esto indica, en nuestra forma de ver, que él lo tomaba prestado de alguien más. ¿Pero de quién? Debido a que Tiktin era de origen alemán y que había realizado en Alemania sus estudios secundarios (nacido en Breslau, se establece en Rumania en 1869, a la edad de 18 años); es en Alemania, creemos, en donde hay que buscar las fuentes primigenias de su ideología gramatical: primero que todo en los manuales de gramática, que habría podido tener en la escuela.

5.2. Ahora bien, el primero que, en nuestra opinión, pudo haber empleado el procedimiento de representación gráfica de relaciones sintácticas en Alemania, fue el teólogo, filósofo y gramático (Johann) Gustav (Friedrich) 
Un precursor desconocido de la sintaxis estructural: H. Tiktin, por Eugenio Coseriu

Billroth (1808-1836) ${ }^{42}$. En su Lateinische Syntax für die obern Klassen gelehrter Schulen, Leipzig (1832, pp. 97 y siguientes), Billroth distingue principalmente la oración que llama «desnuda» («nackt»= nuclear, sin determinaciones suplementarias) y la oración que denomina «vestida» («bekleidet») ${ }^{43}$; este mismo autor desarrolla, brevemente, una teoría de la «vestimenta» ${ }^{44}$ (= determinación) sintáctica y presenta a continuación la «vestimenta» de la oración latina Miltiades, dux Atheniensium, toti Graeciae libertatem paene oppressam in pugna apud Marathonem reddidit ${ }^{45}$, en donde según dice el «cuerpo desnudo» se encuentra constituido únicamente por el sujeto Miltiades y el verbo predicativo reddidit (regresó) (p. 102):

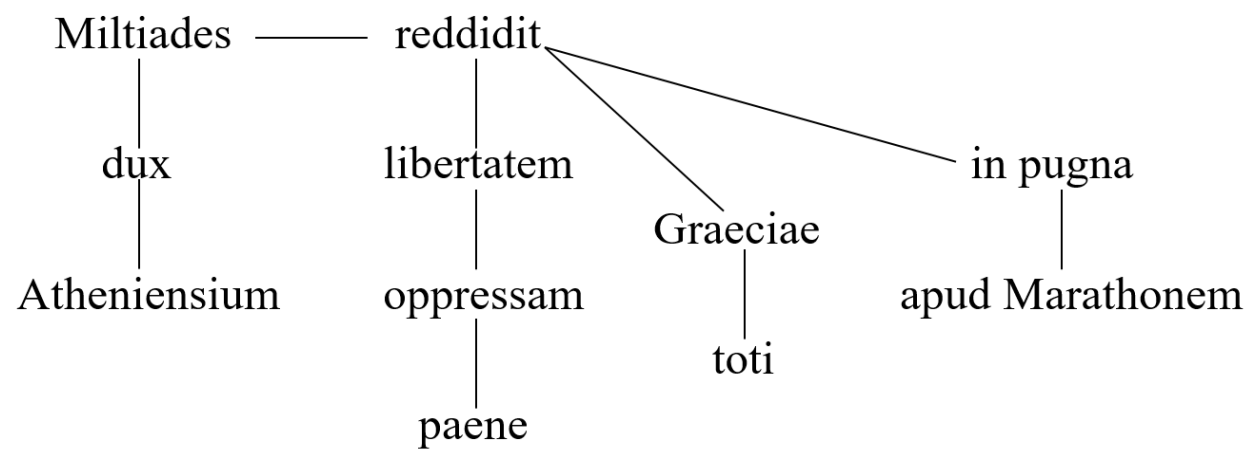

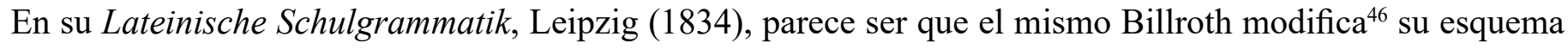
de la siguiente manera:

42. Debemos la indicación a propósito de Billroth a M. M. Sandmann (conferencia realizada en la Universidad de Tübingen en 1970). Pero $c f$. ahora el estudio «La contraction de simples constructions du type S-P», en su recopilación Expériences et critiques, París (1973, p. 82).

43. Esta terminología, muy difundida entre los gramáticos alemanes hasta el último cuarto del siglo xIX, procede, como se sabe, de J. Chr. Adelung.

44. Según Menéndez (1990, p. 259), Kern refutó, por motivos estéticos, la terminología empleada por Billroth, y puso de manifiesto que no es ni adecuado ni de buen gusto analizar los complementos de una oración como si se tratara de camisas, faldas o chalecos (Kern, 1883, p. 96). (N. del T.).

45. «Milcíades, el general ateniense de Grecia, en la lucha por la independencia, casi paralizado regresó por Maratón». (N. del T.).

46. Nosotros decimos «parece ser», porque no hemos podido ver la primera edición de esta obra, la única que apareció cuando Billroth aún vivía. Las ediciones posteriores, a partir de la segunda (1838), fueron publicadas por Friedrich (Theodor E.) Ellendt (17961855). Aquí, citamos según la tercera edición, Leipzig (1848, p. 358). 


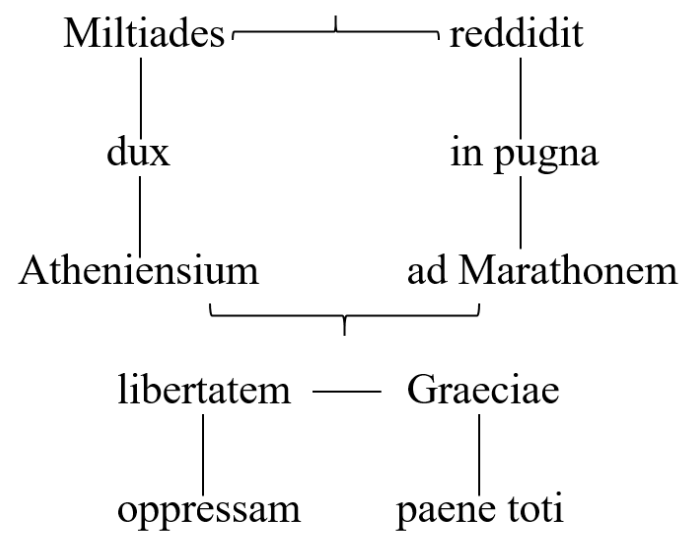

Es decir, que corrige el error que tiene que ver con paene, el cual ya no aparece como determinación de oppressam sino junto a toti (aunque en una relación no precisa), y que, por otra parte, interpreta aparentemente libertatem oppressam paene toti Graeciae como determinación de la frase entera Miltiades dux Atheniensium reddidit in pugna apud Marathonem; además, reemplaza — no se sabe por qué- apud por ad.

Entre Tiktin y Billroth existen, sin embargo, diferencias bastante profundas. Tiktin no subdivide la oración en sujeto y predicado (parte, como se ha visto, del verbo) y emplea una terminología distinta; así, no habla de «vestimenta» sino de «determinación» («determinare»). Por otra parte, es muy poco probable que Tiktin haya conocido la gramática de Billroth en su forma original; $y$, que en la época en la que hubiese podido tenerla como manual en el liceo, ésta ya no contuviese el esquema antes señalado. En efecto, después de la muerte de Ellendt ( $c f$. nota 43), esta gramática fue publicada a partir de la $4^{\mathrm{a}}$ edición (1855) - y, fuertemente modificada desde la 5a edición (1862) — por Moritz Seyffert, quien suprimió de esta el esquema en cuestión así como, por lo demás, toda la sección sobre «la vestimenta» ${ }^{47}$.

5.3. Por el contrario, tenemos fuertes razones para afirmar, con bastante seguridad, que la fuente de Tiktin fue Franz Kern (1830-1894), del cual pudo utilizar las obras ${ }^{48}$ en su enseñanza del alemán en Rumania. De hecho, en esta ocasión, las analogías entre los dos autores son numerosas y sorprendentes, tanto en lo que tiene que ver con la concepción y la terminología gramaticales, como en lo que concierne a la presentación gráfica de las relaciones sintácticas.

En su primera obra gramatical, Die deutsche Satzlehre. Eine Untersuchung ihrer Grundlagen, Berlín (1883, 2a edición, 1888), Kern aún no emplea esquemas. Pero precisa allí, con buenos argumentos, la posición dominante del verbo en la oración (pp. 64 y siguientes) y rechaza, como superfluos (pp. 95 y siguientes), los términos de «desnudo», «vestido»y «vestimenta» (emplea «bestimmt», «Bestimmung»: «determinado», «determinación»),

47. $\quad$ Cf. Dr. Friedrich Ellendt's lateinische Grammatik, bearb. von Dr. Moritz Seyffert, 8. verbesserte Auflage, Berlín (1869). No sabemos exactamente en qué edición Seyffert realizó estas supresiones. Pero es probable que las haya hecho desde la 5 a; los prefacios de la $5^{\mathrm{a}}$, de la $6^{\mathrm{a}}$ y de la $7^{\mathrm{a}}$ edición $(1862,1864,1867)$, reimpresos en la $8^{\mathrm{a}}$, no contienen ninguna indicación al respecto.

48. Aparecidas, sin duda, en una época en la que había abandonado Alemania (para no regresar allí sino hasta 1905). 
Un precursor desconocido de la sintaxis estructural: H. Tiktin, por Eugenio Coseriu

así como la imagen misma del «cuerpo vestido», frente a la cual prefiere la de un árbol con sus ramas y sus hojas ${ }^{49}$. Más aún: Kern propone para esto la justificación explícita de la representación gráfica («graphische Darstellung»), que recomienda, sobre todo con fines prácticos, en lo que concierne a la enseñanza (p. 110). Y emplea una multitud de stemmas, tanto para la oración simple como para la oración compleja, en la obra que publica más adelante, Zur Methodik des deutschen Unterrichts, Berlín (1883, pp. 10, 13, 17, 24-30), y, por lo demás, en su Grundriß der deutschen Satzlehre, Berlín (1884, pp. 30-36, 38, 56). A continuación, tenemos un ejemplo muy característico que representa la oración Eine stolze Krähe schmückte sich mit den ausgefallenen Federn der $\operatorname{Pfauen}^{50}$ (Grundriß, 1884, p. 30):

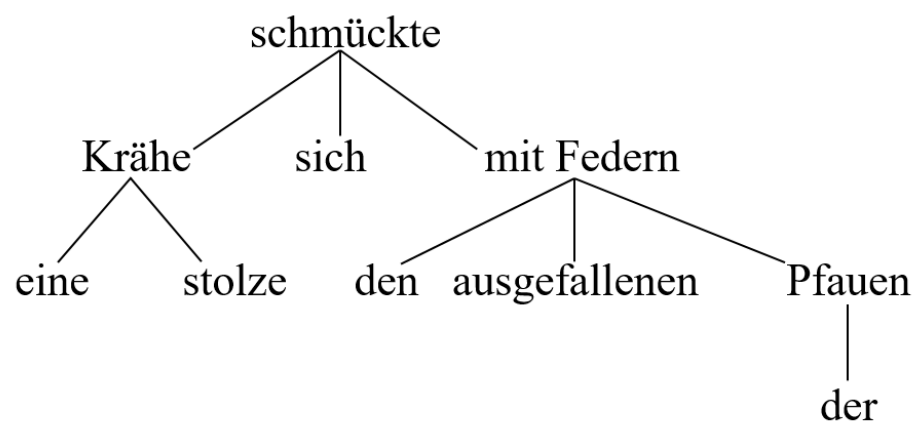

En la obra Zur Methodik, Kern emplea, para la representación gráfica, el término de «Schema». En el Grundriß (aparecido luego en su 2a edición de 1885, y en una 3ª edición de 1896), utiliza el de «Satzbild»; y el procedimiento como tal, lo denomina «anschauliche Darstellung» ( $c f$. la «síntesis figurada» de Tiktin).

6.1. El mismo Kern habla de este procedimiento con la seguridad de ser el primero en proponerlo; como sucede, en particular, en la Satzlehre (1884, p. 110). Pero quizás no se atrevió a relacionarlo con Billroth: en contraste con Tiktin, Kern seguramente pudo conocer la Lateinische Schulgrammatik, y podemos suponer que hubiese guardado un recuerdo más o menos consciente de esta. Podemos notar en efecto, que el esquema empleado por Billroth, a pesar de la división de la oración en sujeto y predicado, es claramente un esquema de «determinación» o de «dependencia», y no de «división» o «adición». Por lo demás, Billroth lo dice explícitamente: «[los elementos añadidos al núcleo de la oración] sind von dem Theile, den sie bestimmen; abhängig, werden von ihm regiert ${ }^{51} »$ (Lat. Syntax, p. 97). Y Kern habla constantemente de "dependencia": «Abhängigkeit» (del término regido, con relación al regente)

6.2. En cuanto a los autores posteriores a Kern, Natanael Beckman se relaciona directamente con el gramático

49. Cf. en particular: «Wie an den Stamm sich Äste, Zweige und Blätter anschließen, so im Satze die finite Verbalform alles Übrige» (p. 101).

50. «Un cuervo orgulloso se adornó con las elegantes plumas de los pavos reales». (N. del T.).

51. «Son de la parte que estos determinan; dependientes, son gobernados por él [núcleo]». (N. del T.). 
Un precursor desconocido de la sintaxis estructural: H. Tiktin, por Eugenio Coseriu

alemán ( $c f .1 .3$.), incluso si este último vuelve a la división de la oración en sujeto y predicado. $C f$. por ejemplo el esquema («karta») que propone de la oración sueca Min äldsta brors hund satt på bakbenen ${ }^{52}$ (Svensk språklära för den högre elementarundervisningen, Stockholm, 1935, p. 226).

Subj. $\longrightarrow$ Pred.
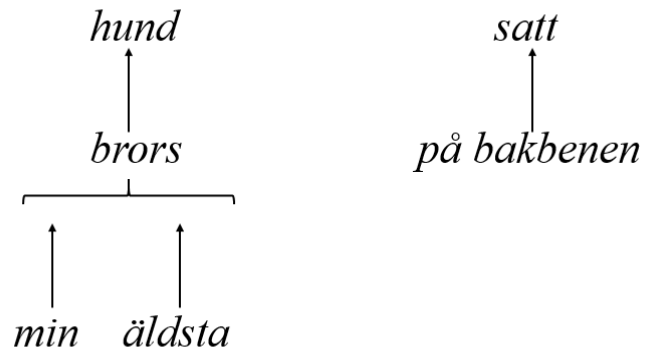

Y puede suceder incluso igual en el caso de Isabel Fry: hemos visto que Jespersen denomina «pictorial analysis», al esquema empleado por esta estudiosa de la gramática; lo que corresponde medianamente al «Satzbild» y al «anschauliche Darstellung» de Kern. Puede observarse, por otra parte, que la mayoría de los autores que han empleado esquemas sintácticos eran autores de manuales y de gramáticas prácticas. Esto nos conduce a suponer también en Tesnière un «recuerdo latente»: no hay que excluir el hecho de que el lingüista francés, excelente conocedor del alemán, haya estudiado esta lengua con la ayuda de las obras de Kern, o que haya por lo menos conocido en su juventud alguna de aquellas.

6.3. Por consiguiente, proponemos a manera de hipótesis (que es importante verificar), en lo que concierne al desarrollo de la sintaxis estructural de representación gráfica, el siguiente árbol genealógico (en el cual indicamos con líneas punteadas las posibles o probables conexiones, pero aún inciertas en la actualidad):

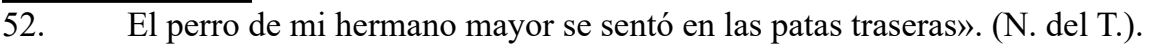




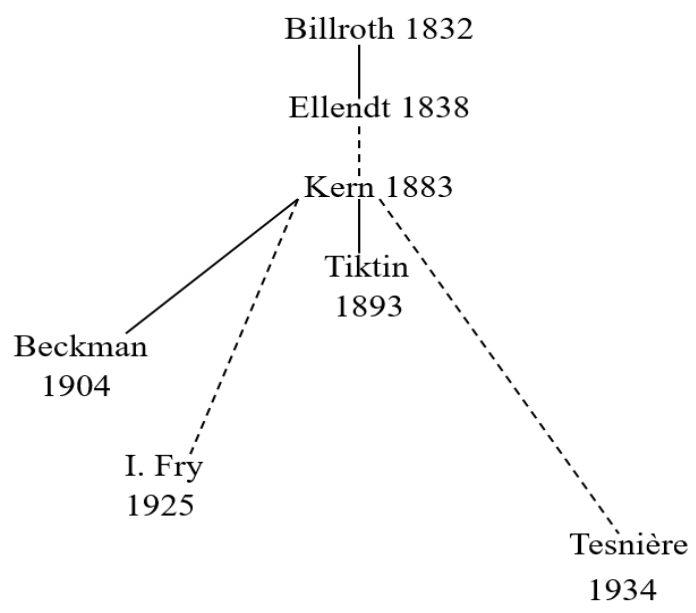

$\mathrm{Y}$ es posible, igualmente, que nos preguntemos si los gramáticos soviéticos mencionados por Tesnière, y los gramáticos norteamericanos promotores del análisis en «constituyentes inmediatos», no podrían también relacionarse, de una u otra forma, directa o indirectamente, con Kern y con la tradición que acabamos de esbozar.

6.4. En todo caso, un hecho mucho más importante desde el punto de vista histórico, nos parece comprobado: la historia de la gramática y de la ideología gramatical es, en gran medida, la de los manuales escolares y de la enseñanza gramatical. Y parece incluso probable que en materia de gramática, los teóricos del lenguaje hayan desarrollado en sus teorías, en más de una ocasión, las ideas que habían adquirido en la escuela.

\author{
Eugenio Coseriu \\ Romanisches Seminar \\ Eberhard Karls Universität Tübingen
}


Un precursor desconocido de la sintaxis estructural: H. Tiktin, por Eugenio Coseriu

\section{Referencias bibliográficas}

\section{Obras citadas por Coseriu}

1. Beckman, N. (1904). Svensk språklära för den högre undervisningen [sic]. Stockholm: A. Bonnier.

2. Beckman, N. (1935). Svensk språklära för den högre elementarundervisningen. Stockholm: A. Bonnier.

3. Billroth, J. G. F. (1832). Lateinische Syntax für die obern Klassen gelehrter Schulen. Leipzig: Weidmann.

4. Billroth, J. G. F. (1834). Lateinische Schulgrammatik. Leipzig: Weidmann.

5. Bloomfield, L. (1933). Language. New York: Henry Holt.

6. Collinder, M. B. (1970). Noam Chomsky und die generative Grammatik. Eine kritische Betrachtung. Uppsala: Acta Societatis Linguisticae Upsaliensis.

7. Fries, Ch. C. (1952). The Structure of English. New York: Harcourt/Brace.

8. Fry, I. (1925). A Key to Language, London: Sidgwick \& Jackson.

9. Gleason, H. A. (1955). An Introduction to Descriptive Linguistics. New York: Henry Holt.

10. Hockett, Ch. F. (1958). A Course in Modern Linguistics. New York: The MacMillan Company.

11. Jespersen, O. (1937). Analytic Syntax. Copenhague: Levin \& Munksgaard.

12. Kern, F. (1883a). Die Deutsche Satzlehre: Eine Untersuchung ihrer Grundlagen. Berlin: Nicolaische VerlagsBuchhandlung.

13. Kern, F. (1883b). Zur Methodik deutschen Unterrichts. Berlin: Nicolaische Verlags-Buchhandlung.

14. Kern, F. (1884). Grundriß der Deutschen Satzlehre. Berlin: Nicolaische Verlags-Buchhandlung.

15. Nida, E. (1946 [1949]). Morphology. The Descriptive Analysis of Words. Ann Arbor: University of Michigan Press.

16. Nida, E. (1959 [1964]). A Synopsis of English Syntax. Oklahoma: Summer Institute of Linguistics of the University of Oklahoma.

17. Rizescu, I. (1971). H. Tiktin, Omul şi opera. Bucarest: Editura ştiinţifică.

18. Sandmann, M. (1973). La contraction de simples constructions du type S-P. In Expériences et critiques. Essais de Linguistique générale et de Philologie romane (pp. 81-94). Paris : Klincksieck.

19. Seyffert, M. (1869). Dr. Friedrich Ellendt's Lateinische Grammatik. Berlin: Weidmannsche Buchhandlung. 20. Tesnière, L. (1934). Comment construire une syntaxe. Bulletin de la Faculté des Lettres de Strasbourg, 12(7), 219-229.

21. Tesnière, L. (1953). Esquisse d'une syntaxe structurale. Paris : Klincksieck.

22. Tesnière, L. (1959). Éléments de syntaxe structurale. Paris : Klincksieck.

23. Tiktin, H. (1891-1893). Gramatica română. Bukarest: Iaşi.

24. Ušakov, Smirnova \& Ščeptova (1929). Učebnaja kniga po russkomu jazyku. Moscú/Leningrado: Gosizdat. 25. Wells, R. S. (1947). Immediate Constituents. Language, 23, 81-117. 
Un precursor desconocido de la sintaxis estructural: H. Tiktin, por Eugenio Coseriu

\section{Obras citadas en el proceso de traducción}

1. Coseriu, E. (1980). Un précurseur méconnu de la syntaxe structurale : H. Tiktin. In Recherches de linguistique : hommage à Maurice Leroy (pp. 48-62). Bruxelles : Éditions de l’Université de Bruxelles.

2. Hudson, R. (2014). Sentence-Diagramming Systems Used in the USA. https://dickhudson.com/grammar-usa/

3. Imrenyi, A. \& Mazziotta, N. (2020). Chapters of Dependency Grammar: A Historical Survey from Antiquity to Tesniere. Amsterdam/Philadelphia: John Benjamins Publishing Co.

4. Mazziotta, N. \& Kahane, S. (2017). To What Extent is Immediate Constituency Analysis Dependency-Based? A Survey of Foundational Texts. Proceedings of the Fourth International Conference on Dependency Linguistics (Depling 2017), (pp. 116-126), Pisa, Italy, September 18-20, 2017.

5. Menéndez, A. G. (1990). Las representaciones: medio para visualizar la estructura de la frase. Philologia Hispalensis, 5(17), 255-263.

6. Osborne, T. (2019). A Dependency Grammar of English: An Introduction and Beyond. John Benjamins Publishing Co.

7. Sarfati, G.-E. \& Paveau, M.-A. (2014). Les grandes théories de la linguistique : De la grammaire comparée à la pragmatique. Paris : Armand Colin.

8. Tesnière, L. (1934). Comment construire une syntaxe. Bulletin de la Faculté des Lettres de Strasbourg, 12(7), 219-229. 